\title{
ERRATUM
}

\section{Total synthesis of the large non-ribosomal peptide polytheonamide B}

Masayuki Inoue, Naoki Shinohara, Shintaro Tanabe, Tomoaki Takahashi, Ken Okura, Hiroaki Itoh, Yuki Mizoguchi, Maiko lida, Nayoung Lee and Shigeru Matsuoka

Nature Chemistry doi:10.1038/nchem.554 (2010); published online: 21 February 2010; corrected online 23 February 2010.

In the version of this Article originally published online, an in-house error led to the incorrect representation of stereochemistry in Figs 1, 2 and 6. These have now been corrected in all versions of the Article. 